\title{
Evolution OF CONSCIOUSNESS I
}

\author{
Richard A. Mould* \\ Department of Physics and Astronomy, State University of New York, Stony Brook, USA \\ Received April 14, 2009; accepted April 29, 2009
}

\begin{abstract}
The existence of a psycho-physical parallelism in humans and other conscious creatures requires an 'interaction' between subjectivity and physiology, where consciousness is as much a casual agent as the molecules and atoms of the system. This interaction is illustrated in the fanciful case of an ancient fish that has a 'painful' experience and a corresponding physiological response. The model gives consciousness properties of both cause and effect that are deterministic; and yet, it reflects our experience of having choice. Experiments are discussed involving PET scans with humans and autoradiographies with rats. They are designed to demonstrate the influence of consciousness on concentrations of narcotics in the brain.
\end{abstract}

Key words: Consciousness; Evolution; Pain; PET

\section{INTRODUCTION}

Most physical and biological scientists say that physiological evolution proceeds along mechanical lines only. It is claimed that the fundamental forces of physics and chemistry combine with chance mutation to select the physiology of a species that will best survive the evolutionary struggle. Consciousness is assumed to have evolved separately. It is understood to have come about through certain physiological make-ups (i.e., certain configurations of neurons) and is somehow a byproduct of fundamental physical forces. However, is not itself said to be a force in nature. It cannot cause things to happen. It is influenced by physiology but it cannot have a reciprocal influence on physiology, although it appears to do so. That appearance is supposedly an illusion with no basis in physics. Consciousness, they say, has no place among the fundamental forces so its imagined influence is a deception.

It is important to recognize the great improbability of this idea. It claims that consciousness has no influence and therefore serves no evolutionary purpose. But if something serves no evolutionary purpose it generally atrophies; or if it doesn't atrophy, it at least ossifies. In any case it does not continue to evolve in functional parallel with the species, achieving higher and higher forms consistent with and appropriate to the physiologi- cal development of the species. For example, an appropriately evolved conscious organism will consciously experience sitting down when its body sits down, and it will consciously experience fear when its body is in physical danger. It would be a miracle if correspondences like that were to occur without good reason without consciousness being interactively engaged with physiology. If a chance mutation did bring about some unrelated and irrelevant conscious phenomenon, there is no reason to believe that it would persist for centuries, developing in parallel with physiology. It is more likely to stage a pointless display for a time and then disappear for lack of purpose. The idea that an inconsequential illusion will persist over time and develop into a reliable mirror of the associated 'physical reality' requires an enormous stretch of imagination. There are many ways that this argument might be stated, but it comes down to two basic parts:

1. Consciousness must have served an evolutionary purpose, for otherwise it would not have survived as it has. It is simply improbable that evolution would produce and nurture a useless appendage to physiology.

2. Consciousness is introduced into a species by genetic mutation for the purpose of supporting physiological evolution. To do that it must interact with physiology in some way, exercising a causal influence of its own.

*E-mail: rmould@notes.cc.sunysb.edu 
To my knowledge the idea expressed here was first put forward by William James who said that the evolution of "appropriate" subjective feelings would be incomprehensible if feelings were biologically redundant (James, 1890).

There are those who characterize consciousness as an airy essence that shrouds physiology without effect. William Clifford says that all matter is accompanied by a rudimentary sentience that flowers into more sophisticated forms of consciousness when matter takes on more sophisticated structural configurations (Clifford, 1886). Also, many in the AI community favor the idea that structural configuration alone (i.e., neurological software) gives rise to consciousness (Hofstadter, \& Dennett, 1981). However, these views do not account for the fact that conscious phenomenon so faithfully reflects the environment in which the body participates. They imagine that an otherwise useless consciousness comes into existence ad hoc, with a mandate to mimic physical reality. But why? Theater like this might be spectacular entertainment, but its underlying thesis is little more than a variation of Leibnitz's doctrine of PreEstablished Harmony. Consciousness with these idle propert-ies is highly unlikely and entirely pointless.

As to the second idea - that consciousness must have an effect on the direction of evolution. If that were not so then evolution would have no way to select against inapp-ropriate conscious experiences. Consciousness might then evolve in a way that diverges arbitrarily from the direction taken by physiology; so a body that is busily engaged in making dinner might possess a psyche that is swimming the English Channel, and a body that is leading in a master's tournament might be subjectively sound asleep. Why would it matter? If consciousness really makes no difference, then its content will make no difference.

I conclude that consciousness does make a difference. It is a causal influence that is not derivable from presently known physical forces. Physics does not now list consciousness as a separate force and has never seriously considered that possibility. This neglect is understandable because the supposed influence, if it does exist, emerges in the most complicated physical system that the universe has yet produced - the conscious living organism. For that reason it would be difficult to untangle consciousness from other influences in order to document its presence and its properties. Most scientists therefore rest on the assumption that there is no such influence inasmuch as they cannot explain that influence. Of course, they can't explain the influence of physiology on consciousness either. Consciousness cannot now be explained as cause or effect. It lies entirely outside the current physics paradigm. Nonetheless, almost everyone believes that physiology influences consciousness without understanding why. The claim here is that the influence of consciousness on physiology is just as believable - although we don't understand that either. When we finally do crack the consciousness/physiology code I think we will find that it is a two-way street like everything else in nature.

I would like to emphasize that I am not talking about the so-called "free will" aspect of consciousness, but rather its "causal" aspect and that only. To say that consciousness has effect without being caused is as puzzling to me as saying that it is caused without having effect. Consciousness is both cause and effect like everything else.

In this paper I suggest an evolutionary mechanism that connects the psychological with the physical, resulting in what von Neumann calls the psycho-physical paralelism (von Neumann, 1955). I will make a specific proposal regarding the relationship of the conscious experience of 'pain' and its physiological counterpart. In the final sections I discuss two experiments that I believe are capable of revealing the influence of consciousness on physiology.

\section{THE PARALELL PRINCIPLE}

The parallel principle states that: physiology and psychology evolve together in any conscious species, where each guides the evolution of the other onto a parallel track.

This parallelism requires some degree of mutual monitoring between the two worlds in order to keep them together on parallel tracks. It means that subjectivity must feed infor-mation back to the underlying physiological system, completing the cycle of influence so the two can evolve together in a complementary way. If there were no feedback, there would be no way for evolution to select against a species that mutates in the direction of inappropriate (i.e., non-parallel) conscious experiences.

\section{A GENERAL SUPPOSITION}

An automaton operates on the basis of a simple stimulus/response sequence that is the sole variable in its evolutionary struggle. But suppose, as a result of mutation, a sequence appears in the form stimulus/consciousness/response. The conscious experience in this sequence does not have to be the sole determinant of the response, but we will allow that it is influential; that is, it will increase or decrease the likelihood of one response or another. Specifically we will say that there exist supportive experiences (like pleasure or lesspain) that always increase a response, and there exist non-supportive experiences (like pain or less-pleasure) that always repressed a response. If the response favored by the newly introduced consciousness is wrong (i.e., if it supports an unfortunate response) then the species will be less likely to survive; but if the favored response is right, then the species will be more likely to survive. In the end, a successful species will have a con- 
scious experience that is supportive of a successful stimulus/response sequence, and this is the signature of a psycho-physical parallelism. This paper focuses initially on pain experiences. We show how these might have been instrumental in establishing the initial psychophysical parallelism in a primitive fish. We then propose an experiment that demonstrates the possible role of 'pleasure' in a contemporary form of the parallelism. In a subsequent paper we will show how other elementary motivational experiences may have come into the evolutionary picture (Mould, 2009). The parallel principle states that:

\section{A FANCIFUL EXAMPLE}

An example that I use in a previous paper involves an ancient fish that brushes against the toxic tentacles of a jellyfish (Mould, 2001a). Assume that the fish has first learned to avoid the jellyfish tentacles without the help of consciousness; so it already has a noceceptive nervous system that favors withdrawal from this toxic contact. Otherwise it would not have survived as long as it has. The nervous system of a fish is very primitive compared with humans. It is dominated by brainstem components that lack a neocortex, and its cortical sensory responses appear to be non-existent since the sensory behavior of a fish is not affected by cortical damage (Bermond, 1997). It is therefore easy to believe that fish are very robot-like creatures in spite of their frequent human-like responses. It is for this reason that I choose a fish to illustrate the possible starting point of conscious intervention.

Pre-conscious contact with the toxic tentacles will lead to two possible responses of the fish that I will label CC or W (continued contact or withdrawal). This will be written

$$
\text { contact leads to }+C C \text { or }++W
$$

where $+C C$ represents the likelihood that fish will remain in 'continued contact' with the tentacles, and $++W$ represents the likelihood that it withdraws from contact. The plus signs indicate the strength of the influence; so the double plus sign in front of $W$ means that the withdrawal response is more probable than continued contact. The pre-conscious fish has apparently evolved sufficiently to prefer $W$ to $C C$, so after a time it will more likely be found withdrawn from the tentacles - hence its survival to date.

As the not-yet-conscious fish evolves further, mutations may occur that cause the withdrawal response to be further strengthened giving

$$
\text { contact leads to }+C C+++W
$$

so $W$ becomes even more probable than $C C$. After a number of mutations of this kind, the $C C$ response might atrophy leaving $W$ as the only possibility. If the mutations mistakenly cause an increase in the probability of $C C$ instead of $W$, then the species will not survive.
Evolution will weed it out. The question then becomes: How will mutations leading to pain consciousness advance the evolution of this fanciful fish? To satisfy the parallel principle, the "pain" associated with the contact state makes W much more probable, so statement 2 will be replaced by

$$
\text { contact resulting in pain leads to }+C C++++W
$$

Our assumption throughout is that the experience of pain will always repress the probability of the response to which it is associated (in this case $C C$ ), and/or it will always support the probability of the opposite response. The difference between statements 1 and 2 is that a mechanical mutation has increased the likelihood of $\mathrm{W}$ in 2; whereas in 3 another kind of mutation introduces 'pain' into the contact state, and that in turn increases the likelihood of $\mathrm{W}$ by an even greater amount. The first mutation in statement 2 appears to be more direct and on that basis it would seem to be preferred. However, at some point the introduction of consciousness must have been the more favored route to survival. So statement 3 must have been preferred for reasons that are not clear at this time. Maybe consciousness is more efficient, allowing it to skip over $+++W$ and go directly to $++++W$ or higher. Maybe a mutation that introduces a conscious experience is more easily achieved than one that tinkers with the noceceptive system alone. The answer to this will probably not be known until we understand how neurological networks introduce consciousness in the first place, and how that change might further affect the subject's response. But whatever the reason for this novel innovation, we can be sure that at some point statement 3 became the preferred path, for it produced a psycho-physical species like our own in which pain is a vital subjective motivator that is well connected with the surrounding objective world.

When I speak of "pain" in this example I do not necessarily refer to the painful experience known to humans. Different creatures might experience pain differently (Nagel, 1974). What is important about pain is its association with a certain kind of evolutionary response illustrated above.

\section{CAUSE AND CHOICE}

Statement 3 also allows the possibility of continued contact, which may be an alternative if the pain is not too overwhelming. In a sophisticated organism $C C$ may be accompanied by other conscious experiences that we might recognize as conflicting motivations - as in the case of an ascetic who makes a point of enduring pain. The subject's experience would then confirm that a conscious choice has been made between continued contact and withdraw. That is, if we are simultaneously stimulated by two conscious motivations in varying degrees, the choice will appear to be a conscious one. However, that choice is basically among casually determined 
probability amplitudes, and there is no reason to believe that it is independent of those probabilities. There is no reason to believe that the choice is a "free" choice, although it may feel that way.

\section{EXPERIMENTS}

The author has proposed experiments involving PET scans with human subjects and/or autoradiographies with rats that are designed to demonstrate the influence of consciousness on concentrations of narcotics in the brain (Mould, 2001b). In both experiments pleasure/pain consciousness is assumed to be correlated with the relative binding of opiates vs non-opiates to receptors in a subject's brain. Two kinds of molecules are injected into the subjects. The first of these are called agonists. These are the opiates (such as morphine) that are recognized by their analgesic and/or euphoric effects when they attach to receptors in the brain. The second are called antagonists that attach to the same opiate receptors; however these produce no pharmacological effects (i.e., no conscious awareness). When these molecules are injected together they compete with one another for attachment to receptors; so they compete with one another to produce a conscious response of either 'pleasure' or 'no pleasure', which is an analog of a 'pain' or 'no pain' competition. We use both hot molecules that are radioactively labeled and cold molecules that are not.

The first experiment involves four PET scans on humans. In the first scan the conscious subject is given a slightly greater than threshold dose of hot agonist and cold antagonist molecues. This is a dose that can just be consciously experienced. The radioactive count CA is recorded in those regions in the brain in which opiate receptors are most numerous. In the second scan the subject is given a comparable dose of hot antagonist and cold agonist molecules; and the radioactive count CAA is recorded in the same regions of the brain. The ratio is given by

$$
r=C_{A} / C_{A A}
$$

The third and fourth scans are identical with the first and second, except that the does in this case are subpharmological (i.e., just below threshold). They are too small to be experienced. This means that consciousness will have nothing to do with the result in the third and fourth scans. The assumption is that this will make a difference in the ratio $r$; so we expect to find that $r$ is greater in the first two scans than in the second two scans.

$$
\begin{aligned}
& r \text { (for the first and second scans above threshold-i.e., the } \\
& \text { more pleasurable scans) } \\
& \quad>r \text { (for the third and fourth scans below threshold) }
\end{aligned}
$$

thereby confirming that consciousness has an influence on physiology. It increases the number of agonist molecules that successfully attach to receptors in competition with antagonist molecules. This assumes that all other influences are equal. Principally this means that the neurological mechanisms involved in the circulation and attachment of agonist or antagonist molecules is linear as we go from unconscious to conscious doses. That is, the number of molecules in an injection does not influence the circulatory or attachment behavior of any one molecule. There is every reason to believe that this linearity holds for these small doses. Of course, an effort must be made to keep all other variables the same throughout the experiment.

The second experiment involves in vivo autoradiographies with rats. Again there are four injections (in four different rats) of mixtures of agonist and antagonist molecules. As before, these are administered in pharmological and subpharmological doses - how-ever that is determined in the case of rats. Each rat is sacrificed so its brain can be sliced and exposed to a film to reveal the concentration of labeled molecules in different brain parts. Again, ratios $r$ in the conscious and unconscious injections are compared with the expectation that the ratio will be greater in the conscious (threshold) case.

\section{OTHER KINDS OF EXPERIENCES}

The fish example and the above experiment deal principally with the pleasure/pain experience and its evolutionary implications. The ideas expressed here will be expanded to other kinds of supportive or non-supportive experiences in Evolution of Consciousness II (Mould, 2009).

\section{REFERENCES}

Bermond, B. (1997). The Myth of Animal Suffering. In: Animal Consciousness and Animal Ethics: Perspectives from the Netherlands. M. Dol (Eds.). Assen: Van Gorcum, pp. 125143.Clifford, W. (1886). Body and Mind. In: Lectures and Essays, L. Stephen and F. Pollock, (eds.). London: Macmillan. Hofstadter, D. \& Dennett, D. (1981). The Mind's I, Fantasies and reflections on self and soul. New York: Basic Books, p. 15.

James, W. (1890). The Principles of Psychology, In: The Works of William James, F. Burkhardt (Ed.), Vol. I, Chap. 5, pp. 141-147. Cambridge, Massachusetts: Harvard University Press: http://psychclassics.yorku.ca/James/Principles/

Mould, R. A. (2001a). The Parallel Principle. arXiv:quant$\mathrm{ph} / 0111096$

Mould, R.A. (2001b). Consciousness and Endogenous State Reduction: Two Experiments." Foundations of Physics Letters, 14, 377-86; arXiv:quant-ph/0106103

Mould, R. A. (2009). Evolution of Consciousness II. Activitas Nervosa Superior, 51, 87-89 (present issue).

Nagel, T. (1974). What is it like to be a bat. Philosophical Review, 83, 435-450

von Neumann, J. (1955). Mathematical Foundations of Quantum Mechanics. Princeton: Princeton University Press, New Jersey, p. 351. 